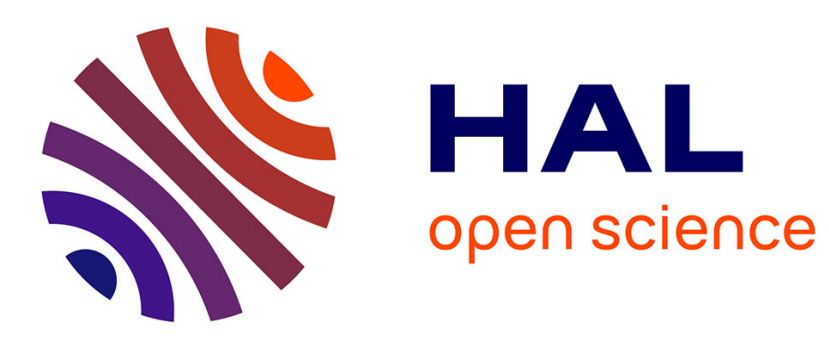

\title{
Capacity Evaluation Framework and Validation of Self-Organized Routing Schemes
}

Hervé Rivano, Fabrice Theoleyre, Fabrice Valois

\section{To cite this version:}

Hervé Rivano, Fabrice Theoleyre, Fabrice Valois. Capacity Evaluation Framework and Validation of Self-Organized Routing Schemes. International Workshop on Wireless Ad-hoc and Sensor Networks, Jun 2006, New-York, United States. pp.779-785, 10.1109/SAHCN.2006.288560 . hal-00371188

\section{HAL Id: hal-00371188 \\ https://hal.science/hal-00371188}

Submitted on 26 Mar 2009

HAL is a multi-disciplinary open access archive for the deposit and dissemination of scientific research documents, whether they are published or not. The documents may come from teaching and research institutions in France or abroad, or from public or private research centers.
L'archive ouverte pluridisciplinaire HAL, est destinée au dépôt et à la diffusion de documents scientifiques de niveau recherche, publiés ou non, émanant des établissements d'enseignement et de recherche français ou étrangers, des laboratoires publics ou privés. 


\section{Capacity Evaluation Framework and Validation of Self-Organized Routing Schemes}

\author{
Herve Rivano \\ CNRS - INRIA Sophia Antipolis \\ 2004, route des Lucioles \\ 06902 Sophia Antipolis Cedex, France \\ Email: Herve.Rivano@sophia.inria.fr
}

\author{
Fabrice Theoleyre, Fabrice Valois \\ INSA Lyon - INRIA Rhone Alpes \\ 21, Avenue Jean Capelle \\ 69621 Villeurbanne Cedex, France \\ Email: \{fabrice.theoleyre, fabrice.valois\}@insa-lyon.fr
}

\begin{abstract}
Assuming a given network topology and a routing protocol, this work is focused on the capacity evaluation of routing protocols based on either a self-organization scheme or a flat approach. To reach this goal, we propose to use linearprogramming formulation to model radio resource sharing as linear constraints. Four models are detailed to evaluate the capacity of any routing scheme in wireless multihops networks. First, two models of fairness are proposed: either each node has a fair access to the channel, or the fairness is among the radio links. Besides, a pessimistic and an optimistic scenarios of spatial re-utilization of the medium are proposed, yielding a lower bound and an upper bound on the network capacity for each fairness case. Finally, using this model, we provide a comparative analysis of some flat and self-organized routing protocols.
\end{abstract}

\section{INTRODUCTION}

Wireless multihops networks are promised to a large spectrum of usages (sensor, ad-hoc or mesh networks) [1]. A fundamental challenge is to provide a tight evaluation of the capacity of a network to conceive adequate applications. Indeed, the radio interferences yield bandwidth sharing mechanisms that are less local and more sophisticated than in wired or cellular networks. Moreover, multihops routes induce strong bandwidth reduction: each forwarded packet creates interferences, burdening other transmissions. [2] was the first work investigating this problem, proposing an asymptotic study. Only few propositions study the trade-off between capacity and network topology.

Routing protocols have been widely studied in wireless multihops networks leading to mainly flat approaches (OLSR, DSR...) Recently, new propositions suggest to apply routing protocols on a self-organization structure [3]. A selforganization creates a hierarchy in the network, based for example on clusters or backbones. However, this approach could reduce the capacity, i.e. the maximum achievable throughput: some radio links are never used and some nodes, acting as leaders, route most of the control traffic. Thus, a fine capacity evaluation is required to quantify this possible drawback. The contribution of this paper is to propose a flexible framework to evaluate the capacity, the network topology and the routes being given.

This work is partly supported by the European Commission, project IST15964. The views given herein represent those of the authors and may not necessarily be representative of the views of the project consortium as a whole.
Our first contribution is to develop four linear programming models of radio resource sharing in 802.11-like networks. Two medium access fairness hypothesis are considered (nodes fairness and links fairness) and we present a pessimistic and an optimistic resource sharing in each case. The models remain valid for any wireless multihops network (e.g. sensor or mesh networks). The second contribution is to compare the capacity of flat and self-organized approaches. This comparative study evaluates the maximum and the max-min capacities achievable by OLSR flat routing scheme [4], VSR [3], and Wu \& Li [5] self-organized approaches.

This paper is organized as follows. Section II summarizes related works about capacity of ad hoc networks. Sections III and IV are dedicated to our linear models of radio resource sharing based on radio interferences and nodes interactions. These models provide lower and upper bounds of the capacity of the network under two hypothesis of medium access fairness. Section V presents the results. We then conclude the article and expose some perspectives.

\section{RELATED WORK}

[2] presents a pioneering work in this area. The authors study the capacity of ad hoc networks, i.e. the maximal achievable throughput. Let $n$ be the number of nodes. The authors demonstrate that in optimal conditions, the global capacity is $O\left(\frac{1}{\sqrt{n}}\right)$. Even in an optimal case, the capacity per node decreases when the number of nodes increases. The authors presented an asymptotic study on the capacity of ad hoc networks independent of any routing protocol, or distributed scheduling technology. This constitutes its strength and its weakness: the capacity evaluation does not model neither a MAC layer behavior, nor the correlation between routing and the capacity. Indeed, the MAC layer regulates the bandwidth sharing. In the same way, the capacity is inherently dependent from the routing protocols: long routes can increase interferences but can also spread the load in the network. In the opposite way, shortest routes will not optimize the capacity [6]. Several articles [7], [8], [9] propose to extend the work [2] to hybrid networks, using the same interference model. Hybrid networks allow to deploy Access Points (AP) in the ad hoc network, and a part of the traffic is sent to Internet through the AP. However, the capacity reaches an asymptotic 


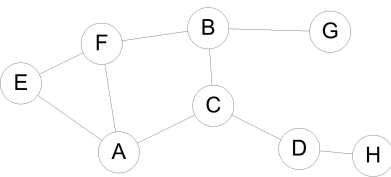

Fig. 1. The 2-Neighborhood of the node C

maximum even when the number of AP becomes arbitrary high.

In [10], the capacity of ad hoc networks is modeled using linear programs (LP). Their work focuses on the capacity estimation for a given network topology and workload. The capacity is modeled as a multicommodity flow problem. However, the complexity of such a formulation resides in the capacity estimation of each link. The authors estimate the maximum capacity in searching sets of links which can convey traffic simultaneously (i.e. they are not interfering). However, their work models an unfair MAC layer.

[11] proposes a scheduling scheme maximizing the throughput from a group of sources to a group of destinations. The authors propose a scheduling of radio links such that no two simultaneously activated links are interfering. A greedy algorithm allocates slots to the different links according to the decreasing euclidean length of the links, this order constituting a sufficient condition of feasibility. However, such an approach could under-estimate the capacity and does not introduce any fairness model.

\section{HYPOTHESIS AND NOTATION}

In this section we introduce the generic form of our linear models for radio resource sharing in wireless IEEE 802.11like networks. The capacity is evaluated for a given network topology and routing protocol. We will discuss later of the fairness assumption.

More precisely, a discrete-event simulation process provides the input data of our models: the network topology, the routes defined by the routing protocol, and the rates of control traffic which is locally broadcasted by each node to its neighborhood. We consider the end to end data transmissions as network flows that induce load on the links they cross, depending directly on the routes created by the routing protocol. The union of the link constraints yields to global network constraints. Besides, the resource sharing is translated in local set of constraints for each node, depending directly on the radio topology. An optimistic and pessimistic models are introduced. Finally, an objective function maximizing the throughput, i.e. estimating the capacity, is used.

In order to develop a linear model of the radio resource sharing, we do some standard assumptions. The radio channel delivers a constant bandwidth and does not corrupt data transmissions. The MAC layer avoids all collisions and the bandwidth required for collision avoidance mechanisms is neglected. A communication is bidirectional: each radio transmission is acknowledged. When control traffic is broadcasted by a node to its neighborhood, all neighbors receive it, which forces 2-neighbors of the source to be silent.

To model interferences, we use the transmitter-receiver model [11]: two links $(A, B)$ and $(C, D)$ can be activated simultaneously if no edge $(X, Y)_{X=\{A, B\}, Y=\{C, D\}}$ exists. The conflict graph could be deducted from the interference model: a node of the conflict graph corresponds to each link in the radio graph. Then, two nodes of the conflict graph have a common edge iif the corresponding radio links are interfering. We will see further how to adapt the following proposition to cope with other interference models.

The following notation is used:

- BW is the available radio bandwidth. This gives the maximum amount of data that can be sent by one terminal.

- $f(p)$ is the throughput of the data sent on the path $p$.

- Let $u$ be a node. $T(u)$ is the total amount of traffic sent by $u$ : $T(u)=\sum_{v \in \Gamma(u)} T(u, v)+T_{c}(u)$ with

- $\Gamma_{k}(u)$ is the $k$-neighborhood of $u$, i.e. the set of the nodes at most $k$ hops far from $u$. $\Gamma_{1}(u)$ is written $\Gamma(u)$ for short. Note that $\forall k, u \in \Gamma_{k}(u)$

- $\Delta_{k}(u)$ is the size of the $k$-neighborhood of $u$ : $\Delta_{k}(u)=\left|\Gamma_{k}(u)\right|$.

- $T(u, v)$ is the unicast traffic on the radio link $(u, v)$ (i.e. $\sum_{p \ni(u, v)} f_{p}$ ).

- $T_{c}(u)$ is the broadcast traffic for control and topology maintenance sent by $u$ to its neighborhood.

\section{MODELS OF RADIO RESOURCE SHARING}

The MAC layer of 802.11-like networks provides a fair access to the channel: when two nodes competes to access the channel, they have the same probability to communicate first. This fairness is quite more complicated to express when the whole network is considered.

In the following, two models of the channel fairness are developed. The first model is node-oriented: nodes access the channel following a uniform distribution. The second model is link-oriented: the point-to-point communication between neighbors have the same probability to access the medium. For each model of fairness, we propose a pessimistic radio resource sharing computing a lower bound on the capacity, and an optimistic radio resource sharing LP yielding an upper bound.

\section{A. Node-oriented fairness}

1) A pessimistic radio resource sharing scenario: A lower bounding linear program is obtained when local radio resource sharing constraints model a pessimistic behavior of the MAC layer. The worst case for contention is when a node broadcasts control traffic. Indeed these packets have to be received by all its neighbors, which requires that all 2-neighbors of the source keep silent. This silence includes refusing any incoming communication request, since it requires to send an acknowledgment for the received packet.

Thus, a pessimistic resource sharing does not distinguish broadcast and unicast communications. This guarantees that data communications are successful, but overestimates interferences.

The node-oriented fairness is modeled by an equal share of the bandwidth among 2-neighboring nodes. Therefore, the 
available bandwidth to a node is constrained by the cardinality of each 2-neighborhood set it belongs to.

This pessimistic MAC local behavior can be described by linear constraints as follows. Suppose we consider a node $c$, called the center of its 2-neighborhood.

- The radio bandwidth is uniformly distributed between all nodes in potential contention: the center $c$ and its whole 2-neighborhood, $\Gamma^{2}(c)$ :

$$
\forall c, \forall u \in \Gamma^{2}(c), T(u) \leq \frac{B W}{\Delta_{2}(c)}
$$

Note, that for each center $c$, a set of $\Delta_{2}(c)^{2}$ equations is given. In consequence, the capacity $T(u)$ allocated to a node $u$ is constrained on average by $\Delta_{2}(u)$ equations (one centered in each 2-neighbor).

- In the capacity allocated to one node, all the control traffic and the unicast transmissions must be scheduled. Additionally, a node allocates an equal capacity to each of the communication links with its neighbors:

$$
\forall u, \forall v \in \Gamma(u)-\{u\}, T(u, v) \leq \frac{T(u)-T_{c}(u)}{\Delta(u)-1}
$$

The equation (2) models that each node manages its available bandwidth, while (1) models that the radio medium capacity is shared among the nodes.

Finally, two nodes can send data simultaneously only if they are sufficiently distant, at least 3 hops, and a low loaded node is given the same bandwidth than a fully loaded one. This set of local constraints yields LP 1, whose solutions lower bound the total amount of traffic supported by the network.

Linear Program 1 (Pessimistic model): Maximize

Objective function on $\mathrm{P}$

\section{Subject to}

Equation set (1)

Equation set (2)

Traffic management for $\mathrm{p}$ node $\mathrm{c}$, the center

$\forall$ node $u$

$\forall$ path $\mathrm{p}$

2) An optimistic radio resource sharing scenario: The pessimistic radio resource sharing model leads to over-estimate the interferences. Some simultaneous communications could be possible in a more realistic protocol, but are forbidden by our previous model. Hopefully, many protocols, like IEEE 802.11, achieve a better repartition. For example, in fig.1, the transmissions $(\mathrm{A} \rightarrow \mathrm{E})$ and $(\mathrm{B} \rightarrow \mathrm{G})$ can be active simultaneously, because the packets are not to be understood by $C$.

Indeed, a set of communications that can occur simultaneously is an independent set of the conflict graph. An optimistic resource sharing model would consider that a maximal independent set of the conflict graph is active. That would upperbound the capacity of the network since the existence of a distributed process realizing such an optimal call scheduling is assumed. Furthermore, describing all the maximal independent sets of a graph is an NP-hard problem. Our optimistic model avoids this problem by considering the constraints on simultaneous communications at a local view point. In other words,

\footnotetext{
${ }^{2}$ One equation for each 2-neighborhood sets it belongs to
}

the model assumes that the local maximal independent sets can be combined into a global one. This obviously weakens the constraints, yielding an upper bound on the global capacity of the network.

Suppose that the channel is free. When one neighbor of the center, $u$, sends a packet to $v$, all neighbors of $v$ has to keep silent. When $u$ finishes the transmission, $v$ sends a MAC acknowledgment, blocking all neighbors of $u$. Suppose that another node $u^{\prime}$ wants to send a packet. If $u^{\prime}$ is neither neighbor of $u$ nor of $v, u^{\prime}$ can initiate a communication. Nevertheless, for the communication to succeed, the destination has to be outside the neighborhood of $u$ or $v$. This behavior is strongly linked to the combinatorial concept of independent set.

Indeed, such a contention-free communication set is an independent set, maximal for inclusion, of the graph $L_{1,2}\left(\mathcal{L}\left(G_{c}\right)\right)$, defined as follows:

- $G_{c}$ is the graph of the 2-neighborhood of $c$.

- $\mathcal{L G}=\mathcal{L}\left(G_{c}\right)$ is the linegraph of $G_{c}$, that is the graph with one vertex per arc of $G_{c}$, and a link between any two vertices whose corresponding arcs are adjacent.

- $L_{1,2}(\mathcal{L G})$ is the graph with the same vertices as $\mathcal{L G}$, and a link between any two 1-neighboring or 2-neighboring vertices. $L_{1,2}(\mathcal{L G})$ is the conflict graph with the transmitterreceiver interference model.

Independent vertices (i.e. pairwise non adjacent vertices) of $L_{1,2}\left(\mathcal{L}\left(G_{c}\right)\right)$ correspond to contention-free communications. An inclusion-wise maximal independent set is therefore an inclusion-wise maximal set of communications that can be activated simultaneously.

Eventually, the MAC layer achieves a fair sharing of the bandwidth among the maximal independent sets. Fairness of bandwidth sharing among the nodes must be respected. Let $B W(I)$ be the bandwidth given to the independent set $I \in$ $\mathcal{I}$, the set of all maximal independent sets of $L_{1,2}\left(\mathcal{L}\left(G_{c}\right)\right)$. $B W(I)$ is proportional to $P(I)$, the probability of $I$ to be selected. $P(I)$ depends on the links it is composed of and from their order of activation. The bandwidth in the neighborhood of $c$ is shared as follows:

$$
\begin{array}{r}
B W(I)=P(I) \cdot\left(B W-T(c)-\sum_{u \in \Gamma(c)-\{c\}} T_{c}(u)\right) \\
\Rightarrow B W \geq T(c)+\sum_{I \in \mathcal{I}} B W(I)+\sum_{u \in \Gamma(c)-\{c\}} T_{c}(u)
\end{array}
$$

The total bandwidth allocated to a communication link $(u, v)$ is the sum of the bandwidth allocated to each independent set including $(u, v)$ :

$$
\begin{gathered}
T(u, v) \leq \sum_{I \ni(u, v)} B W(I) \\
T(u, v) \leq\left(B W-T(c)-\sum_{x \in \Gamma(c)-\{c\}} T_{c}(x)\right) \cdot \sum_{I \ni(u, v)} P(I)
\end{gathered}
$$

Moreover, $\sum_{I \ni(u, v)} P(I)$ is exactly the probability for the communication link $(u, v)$ to be activated by the channel. This quantity is hence denoted $P(u, v)$ in the following. 


$$
\forall(u, v) \in \Gamma^{2}(c)-\{c\},
$$

$$
T(u, v) \leq\left(B W-T(c)-\sum_{x \in \Gamma(c)-\{c\}} T_{c}(x)\right) \cdot P(u, v)
$$

Unfortunately, on arbitrary network topologies, $P(I)$ and $P(u, v)$ can not be computed unless the whole set $\mathcal{I}$ is known, and $\mathcal{I}$ has an exponential size. We therefore build a stochastic estimation of $P(u, v)$, denoted $f r e q(u, v)$ in the following.

These frequencies $\operatorname{freq}(u, v)$ must absolutely take into account the fairness among the nodes. Hence, we propose the following algorithm to construct an independent set:

- While at least one not blocked node exists, take randomly one, say $u$

- choose randomly one neighbor $v$ of $u$ which is not blocked

- If $v$ exists, activate the communication $(u, v)$ and mark all the neighbors of $u$ and $v$ as blocked

- else, mark $u$ as blocked

If this algorithm is repeated $n$ times, $f r e q(u, v)$ is equal to the proportion of the cases where the link $(u, v)$ was selected. Note that each link is directed: the link $(u, v)$ will not receive the same amount of traffic as $(v, u)$.

In order to complete the model, control and topology maintenance traffic generated by the routing algorithms is to be completely taken into account. The equation set (7) models that when a neighbor of the center $c$ emits control traffic, $c$ has to stop any radio activity. On the other hand, the 2-neighbors of $c$ will include their control traffic into their allocated bandwidth.

The bandwidth is distributed per link: a node is not allowed to distribute locally the traffic to each of its link. Indeed, the capacity allocation takes into account the specificities of each link, and particularly the fact that several links can send information simultaneously. If the node chooses itself to redistribute the capacity of an unloaded link to another of its links, the interference constraints could be violated. Such a behavior must be avoided.

The last optimistic aspect of this model is that the combination of the local constraints might not yield a feasible share of the global capacity. As a matter of fact, the union of the local independent sets might not be a global independent set. In other words, the global constraints are stronger that the union of the local ones. The linear program 2 neglects this fact, yielding an upper bound on the global capacity of the network.

Linear Program 2 (Optimistic model):

\section{Maximize}

Equation set (7)

Traffic management for $\mathrm{p}$
Objective function on $\mathrm{P}$

Subject to

$\forall$ link $(u, v) \in E$

$\forall$ path $\mathrm{p}$

\section{B. Link-oriented fairness}

The following section is dedicated to presenting an evolution of the previous linear programs that models a fair allocation of bandwidth among radio links. Consequently, the nodes which have more active links will receive more bandwidth and bottlenecks could be potentially avoided.

In the following, we use the following notations.

- $\mathcal{L G}$ : the linegraph of $G$

- $\gamma_{k}$ : the k-neighborhood in $\mathcal{L G}$ of a link $e$ in $G$. Each link is directed.

- $\delta_{k}:\left|\gamma_{k}\right|$

1) A pessimistic resource sharing scenario: This model operates a pessimistic allocation of the bandwidth among the links. More precisely, when focusing on a link $e$, the bandwidth is distributed among the set of links which are neighbors of $e$ in the conflict graph. In our case, the transmitter-receiver interference model induces that a link conflicts with each link in $\gamma_{2}(e)$. The capacity is then uniformly distributed to the links as follows.

$\forall e \in E, \forall f \in \gamma_{2}(e), T(f) \leq \frac{B W-\sum_{(u, x) \in \gamma_{2}(e)} T_{c}(u)}{\delta_{2}(e)}$

Finally, we obtain the following linear program LP 3.

Linear Program 3 (Pessimistic model):
Maximize

Equation set (8)

Traffic management for $\mathrm{p}$
Objective function on $\mathrm{P}$

Subject to

link e

$\forall$ path $\mathrm{p}$
Note the lower bounds with link-oriented and node-oriented fairness are not comparable. A different behavior of the MAC layer is modeled. Thus the capacity of the network depends on the protocol chosen to allow concurrent access to the medium.

2) An optimistic resource sharing scenario: The upper bound with link-oriented fairness knows less modifications. The only change relies in the probability for a link to be activated, and therefore the capacity that is allocated to each link. Indeed, the algorithm computing the frequencies, $\operatorname{freq}(u, v)$, capacity allocates to each link in the 2-neighborhood of one node must be changed into the following one:

- While at least one not blocked link exists, take randomly one, say $(u, v)$

- Mark as blocked any link $(x, y)$ such that $(a, b)$ and/or $(b, a)_{a \in\{x, y\}, b \in\{u, v\}}$ exists (i.e. $(x, y)$ and $(u, v)$ are interfering).

If this algorithm is repeated $n$ times, freq $(u, v)$ equals the proportion of the cases where the link $(u, v)$ has been selected. Remind that the links are directed: the link $(u, v)$ may not receive the same amount of bandwidth as $(v, u)$. However, fairness among links is respected.

The remaining description of the upper bound remains unchanged. The linear program LP 2 keeps on holding, with the new values of $\operatorname{freq}(u, v)$. 


\section{Flexibility of the models}

All previous models where detailed with the transmitterreceiver model to represent interferences. However, our approach is generic and applies to any arbitrary interference model. One would only need to change the definition of $\Gamma_{k}(e)$ and $\gamma_{k}(e)$ sets into "sets of nodes (resp. links) interfering with node (resp. link) $e$ ". One can therefore use these models as a framework for benchmarking interference models.

In the following, our models assume the transmitter-receiver interference model and allows to compare the capacity of flat and self-organized routing protocols.

\section{Objective functions}

The previously defined linear programs describe how the radio resource is shared among nodes and links. The capacity of a network is then the maximum quantity of information that can be transported by the network. In the following, we define two notions of network capacity, and therefore two objective functions. The first one is the standard sum of transported traffics while the second one provides a user-wise throughput guaranty.

1) Max-Sum objective: The capacity is often described as the maximal sum of throughput achievable in the network, as follows.

$$
\operatorname{Max} \sum_{p \in \mathcal{P}} f(p)
$$

This objective is global and does not address the distribution of the throughput among the flows. Consequently, the less interfering flows are privileged and some flows will be null because they consume too many resources. In practice, the positive flows are almost only single hop flows. There are almost no application where this notion of capacity is relevant. However, this formulation gives an upper bound of the global achievable capacity.

2) Max-Min objective: A telecommunication operator deploying such a network could be interested in providing a guarantee on the minimum bandwidth available to each user. In such a case, the objective is to maximize this flow-wise capacity as follows.

$$
\operatorname{Max}\left(\operatorname{Min}_{p \in \mathcal{P}} f(p)\right)
$$

Of course, this strong guaranty decreases the global achievable throughput. Nevertheless, such a notion of capacity is relevant to numerous applications in ad hoc and hybrid networks.

\section{RESULTS}

Our objective is to estimate the capacity of different routing protocols based on either a self-organized scheme or a flat approach. To reach such a goal, the behavior of some routing protocols were simulated with OPNET Modeler. More precisely, topologies from 20 to 60 nodes with an average degree of 10 nodes were generated. Then, OLSR (relevant to represent flat routing protocols), $\mathrm{Wu} \& \mathrm{Li}$ (a backbone is built, and all the routing is done through this backbone) and VSR (routing protocol where the backbone is only used for the control traffic) were implemented and simulated on these topologies.

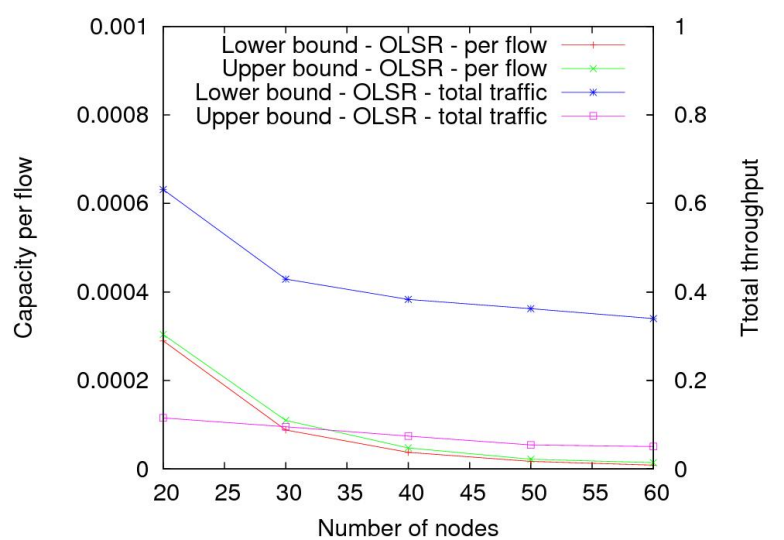

Fig. 2. Comparison of the objective functions in an ad-hoc flat network (link-oriented fairness)

The computed routes and the overhead were directly extracted from the simulations to be used in our LP formulation. We chose to model an ad-hoc traffic pattern: a node has a route toward each other node in the network $(n(n-1)$ routes at all).

First, the general evolution of the capacity in MANET is evaluated with the link-oriented fairness and the max-min objective (fig. 2). With a flat routing protocol, the capacity per flow decreases when the number of nodes increases: the number of flows grows (there exist $n(n-1)$ routes), creating potentially more contention. Consequently, the bandwidth allocated to each flow will surely decrease, corroborating the results of [2]. Oppositely, the total throughput sent across the network remains constant: many flows will with high probability pass through the center of the network since shortest paths are used, creating a bottleneck. Finally, we can note that the optimistic and the pessimistic models present a very close capacity.

The capacity of ad-hoc networks according to different routing protocols is evaluated with the max-min objective and with a link-oriented fairness bandwidth sharing (fig. 3). The flat routing protocol presents the highest capacity: shortest routes in the initial network limit the route length, and consequently present less interferences. VSR presents the same capacity as OLSR: the backbone is only used for control traffic, and although not shortest routes are used, the impact on the capacity is restricted. Oppositely, $\mathrm{Wu} \& \mathrm{Li}$ presents the lowest capacity: it computes shortest routes through the backbone, lengthening the average route.

In a second time, the capacity is evaluated with the nodeoriented fairness bandwidth sharing (fig. 4). The capacity of OLSR and VSR is very slightly impacted by a different fairness model. On the other hand, the capacity presented by $\mathrm{Wu} \&$ Li decreases: all the nodes receive the same bandwidth although backbone nodes carry most traffic. Consequently, a bottleneck in the backbone appears.

Finally, we maximize the global network throughput using the max-sum objective (fig. 5). Some flows are authorized to receive more traffic than others, discriminating the flows which create many interferences(i.e. multihops flows). Consequently, less flows are active simultaneously, and the center of the network constitutes no more a bottleneck: the capacity is stable or increases with the optimistic bandwidth sharing. 


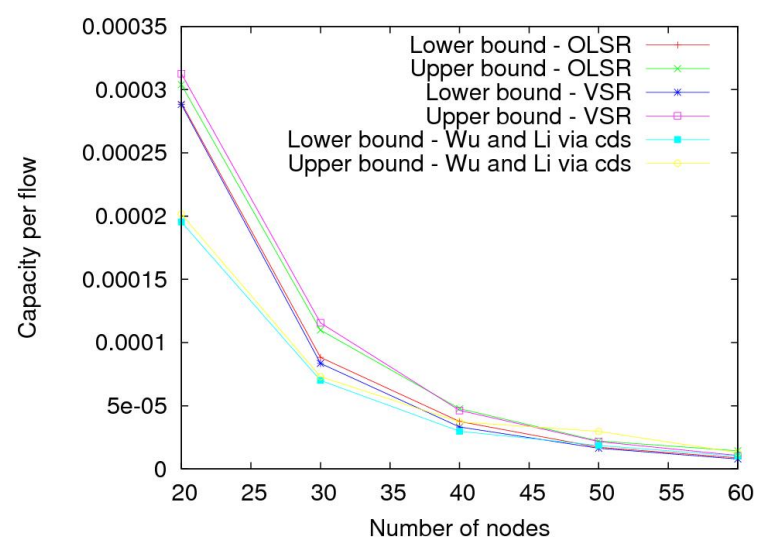

Fig. 3. Capacity with the max-min objective (link-oriented fairness)

Oppositely, the pessimistic resource sharing tends to overestimate the interferences, limiting the spatial re-utilization in small networks. Besides, we can remark that OLSR and VSR provide a very close capacity, whatever the objective function is. On the other hand, Wu \& $\mathrm{Li}$ routing performs much lower

\section{CONCLUSION AND FUTURE WORK}

The goal of this work is to study the capacity of routing protocols based on virtual structure or flat approaches. Thus, we propose a generic capacity evaluation framework used to benchmark routing protocols and to validate the selforganization approach in routing protocols.

Our capacity evaluation framework is made of four linear programs. A set of linear constraints model the local radio resource sharing principles. These local constraints are combined so as to define a global behavior of the network. We provide linear models for two MAC layer fairness hypotheses. One assumes that the probability to access the radio channel is uniformly distributed among the nodes, while the other one assumes a uniform distribution of medium access on the radio links. For each of these settings, we propose a pessimistic and an optimistic scenarios of spatial-reutilization of the medium, hence providing an upper and a lower bound.

Using these models, we compare the capacity provided by a flat routing protocol (OLSR) and two routing protocols based on virtual backbones. The results highlight that the capacity provided by self-organization schemes keeps very close to the performances of standard flat protocols, if the virtual structure

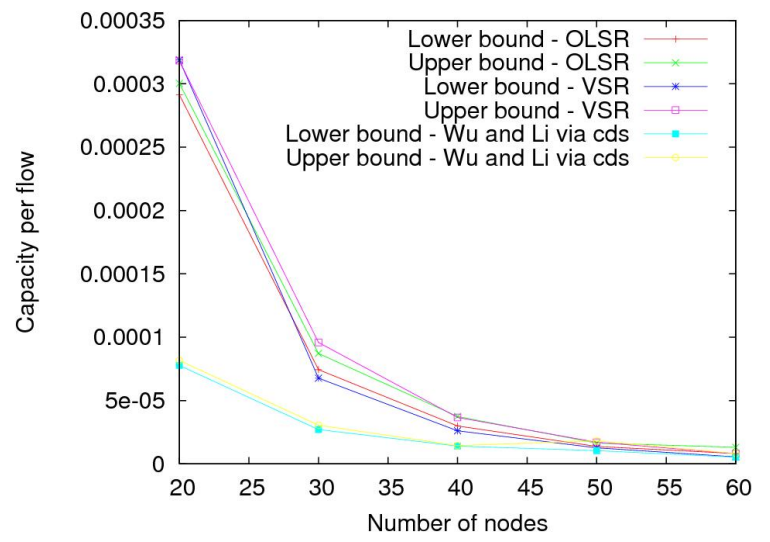

Fig. 4. Capacity with the max-min objective (node-oriented fairness)

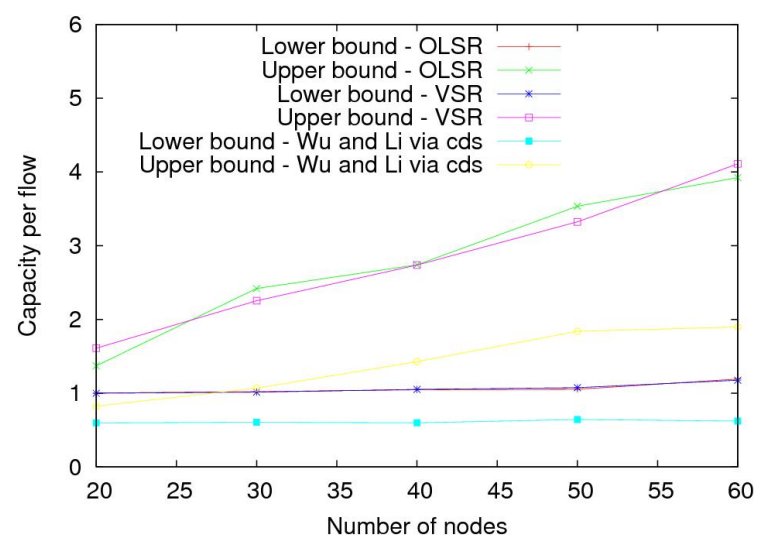

Fig. 5. Capacity with the max-sum objective (link-oriented fairness)

is used only for transporting the control traffic.

More benchmarking have to be done, including other flat routing protocols (AODV, DSR, DSDV...), even though we conjecture that there performances should theoretically be similar to those of OLSR since they all compute almost shortest paths. Multi-path routing protocols should also be studied. Besides, this work is a first step toward the design of routing protocols maximizing the capacity.

\section{REFERENCES}

[1] G. Mario, "From battlefields to urban grids: New research challenges in ad hoc wireless networks," Pervasive and mobile computing, vol. 1, no. 1, pp. 77-93, March 2005.

[2] P. Gupta and P. R. Kumar, "The capacity of wireless networks," IEEE Transactions on Information Theory, vol. 46, no. 2, pp. 388-404, 2000.

[3] F. Theoleyre and F. Valois, "Virtual structure routing in ad hoc networks," in ICC. Seoul, Korea: IEEE, May 2005.

[4] T. Clausen and P. Jacquet, "Optimized link state routing protocol (OLSR)," IETF, RFC 3626, October 2003.

[5] J. Wu and H. Li, "A dominating-set-based routing scheme in ad hoc wireless networks," Telecommunication Systems Journal, vol. 18, no. 1-3, pp. 63-83, September 2001.

[6] D. S. J. De Couto, D. Aguayo, B. A. Chambers, and R. Morris, "Performance of multihop wireless networks: shortest path is not enough," ACM SIGCOMM Computer Communication Review, vol. 33, no. 1, pp. 83-88, January 2003.

[7] U. C. Kozat and L. Tassiulas, "Throughput capacity of random ad hoc networks with infrastructure support," in MOBICOM. San Diego, USA: ACM, September 2003, pp. 55-65.

[8] B. Liu, Z. Liu, and D. Towsley, "On the capacity of hybrid wireless networks," in INFOCOM, vol. 3. San Francisco, USA: IEEE, April 2003, pp. $1543-1552$.

[9] A. Zemlianov and G. De Veciana, "Capacity of ad hoc wireless networks with infrastructure support," IEEE Journal on Selected Areas in Comunications, vol. 23, no. 3, pp. 657-667, March 2005.

[10] K. Jain, J. Padhye, V. Padmanabhan, and L. Qiu, "Impact of interference on multi-hop wireless network performance," in MOBICOM. San Diego, USA: ACM, September 2003, pp. 66-80.

[11] A. Kumar, M. Marathe, S. Parthasarathy, and A. Srinivasan, "Algorithmic aspects of capacity in wireless networks," in SIGMETRICS, vol. 33. Banff, Canada: ACM, 2005, pp. 133-144. 